\title{
Analytical solution to a hydrodynamic model in an open uniform reservoir
}

\author{
Kaboon Thongtha ${ }^{1,2^{*}}$ and Jaipong Kasemsuwan ${ }^{1}$
}

\author{
"Correspondence: \\ kaboon.t@gmail.com \\ 'Department of Mathematics, \\ Faculty of Science, King Mongkut's \\ Institute of Technology Ladkrabang, \\ Bangkok, 10520, Thailand \\ ${ }^{2}$ Centre of Excellence in \\ Mathematics, CHE, Si Ayutthaya \\ Road, Bangkok, 10400, Thailand
}

\begin{abstract}
The hydrodynamic model is used to determine the water wave flow. In this research, a nondimensional form of a two-dimensional hydrodynamic model with generalized boundary condition $g(x, t)$ and initial conditions for describing the elevation of water wave in an open uniform reservoir is proposed. The separation of variables method with mathematical induction is employed to find an analytical solution to the model. An example of flow calculations in an open uniform reservoir is also demonstrated.
\end{abstract}

MSC: 35L05; 35L04; 35L40

Keywords: separation of variables method; hydrodynamic model; open uniform reservoir; water elevation

\section{Introduction}

In [1] and [2], the finite element method was used to solve the water pollution models. In literature, several mathematical models need the data of water flow, while the velocity and elevation of water flow are provided by the hydrodynamic model. In [3], the finite difference method was used to solve the hydrodynamic model with constant coefficients in the closed uniform reservoir.

In [4], an analytical solution to the hydrodynamic model in a closed uniform reservoir was proposed. In [5], the Lax-Wendroff finite difference method was also proposed to approximate the water elevation and water flow velocity. However, the analytical solution to the hydrodynamic model in an open reservoir has not been considered.

An open uniform reservoir is supplied by the outer water wave as shown in Figure 1. In the former studies, the elevation of water wave (tidal elevation) can be found only by numerical approximations. The purpose of this research is to derive an analytical solution to a model. An analytical solution is needed for comparison with the approximated solutions of the flow. Hence, the analytical solution becomes a benchmark for any related numerical approximations.

\section{A nondimensional form of a hydrodynamic model}

The continuity and momentum equations are governed by the hydrodynamic behavior on the reservoir. We average the equations over the depth, discarding the term due to Coriolis parameter, shearing stresses and surface wind. We introduce the well-known two-

(c) The Author(s) 2017. This article is distributed under the terms of the Creative Commons Attribution 4.0 International License (http://creativecommons.org/licenses/by/4.0/), which permits unrestricted use, distribution, and reproduction in any medium, provided you give appropriate credit to the original author(s) and the source, provide a link to the Creative Commons license, and indicate if changes were made. 


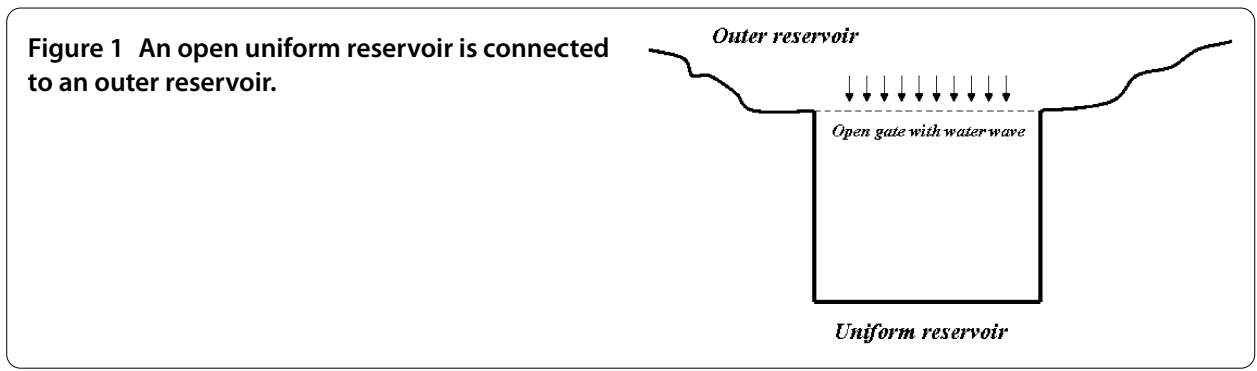

dimensional shallow water equations $[2,5]$ as follows:

$$
\begin{aligned}
& \frac{\partial \zeta}{\partial t}+\frac{\partial}{\partial x}[(h+\zeta) u]+\frac{\partial}{\partial y}[(h+\zeta) v]=0, \\
& \frac{\partial u}{\partial t}+g \frac{\partial \zeta}{\partial x}=0 \\
& \frac{\partial v}{\partial t}+g \frac{\partial \zeta}{\partial y}=0
\end{aligned}
$$

where $h(x, y)$ is the depth measured from the mean water level to the bed of the reservoir, $\zeta(x, y, t)$ is the elevation from the mean water level to the temporary water surface or the tidal elevation, $g$ is the acceleration due to gravity, and $u(x, y, t)$ and $v(x, y, t)$ are the velocity components in $\mathrm{x}$ and $\mathrm{y}$ directions, respectively, for all $(x, y, t) \in[0, l] \times[0, l] \times[0, \mathcal{T}]$. We assume that $h$ is a constant and $\zeta \ll h$. Then Eqs. (1)-(3) lead to

$$
\begin{aligned}
& \frac{\partial \zeta}{\partial t}+h \frac{\partial u}{\partial x}+h \frac{\partial v}{\partial y} \doteq 0, \\
& \frac{\partial u}{\partial t}+g \frac{\partial \zeta}{\partial x}=0, \\
& \frac{\partial v}{\partial t}+g \frac{\partial \zeta}{\partial y}=0 .
\end{aligned}
$$

We will consider the equation in a dimensionless problem by letting $U=u / \sqrt{g h}, V=$ $v / \sqrt{g h}, X=x / l, Y=y / l, Z=\zeta / h$ and $T=t \sqrt{g h} / l$. Substituting them into Eqs. (4)-(6) leads to

$$
\begin{aligned}
& \frac{\partial Z}{\partial T}+\frac{\partial U}{\partial X}+\frac{\partial V}{\partial Y}=0 \\
& \frac{\partial U}{\partial T}+\frac{\partial Z}{\partial X}=0 \\
& \frac{\partial V}{\partial T}+\frac{\partial Z}{\partial Y}=0
\end{aligned}
$$

In order to solve Eqs. (7)-(9) in $\Omega \times[0, T]$, where $\Omega=(0,1) \times(0,1)$, for convenience using $u, v, d$ for $U, V$ and $Z$, respectively [5], we get

$$
\begin{aligned}
& \frac{\partial d}{\partial t}+\frac{\partial u}{\partial x}+\frac{\partial v}{\partial y}=0, \\
& \frac{\partial u}{\partial t}+\frac{\partial d}{\partial x}=0, \\
& \frac{\partial v}{\partial t}+\frac{\partial d}{\partial y}=0 .
\end{aligned}
$$




\subsection{Initial and boundary conditions for an open uniform reservoir}

The initial conditions of Eqs. (10)-(12) are assumed to be $u=0, v=0, d=f_{1}(x, y)$ and $\frac{\partial d}{\partial t}=$ $f_{2}(x, y)$. The boundary conditions of the model in an open uniform reservoir are assumed to be $v=0, \frac{\partial u}{\partial x}=0$ at the planes $x=0$ and $x=1 . u=0, \frac{\partial v}{\partial y}=0$ at the planes $y=0 . u=0$, $\frac{\partial v}{\partial y}=0, d(x, 1, t)=g(x, t)$ and $\frac{\partial d}{\partial y}=0$ at the planes $y=1 . d=0$ on $\partial \Omega \backslash\{(x, y) \in \partial \Omega: y=1\}$.

\section{An analytical solution}

Eqs. (10)-(12) can be written as a non-conservation form of equation, as a single equation,

$$
\frac{\partial^{2} d}{\partial t^{2}}=\frac{\partial^{2} d}{\partial x^{2}}+\frac{\partial^{2} d}{\partial y^{2}}
$$

subject to the initial conditions $d(x, y, 0)=f_{1}(x, y)$ and $\frac{\partial d}{\partial t}=f_{2}(x, y)$. The boundary conditions are $d=0$ at $\partial \Omega \backslash\{(x, y) \in \partial \Omega: y=1\}, d(x, 1, t)=g(x, t)$ and $\frac{\partial d}{\partial y}=0$ at the plane $y=1$. It is readily verified that the general solution can be written by the separation of variables technique

$$
d(x, y, t)=X(x) Y(y) T(t)
$$

subject to the boundary conditions $X(0)=X(1)=0, Y(0)=Y^{\prime}(1)=0$ and $Y(1)=g(x, t)$. Due to the eigenvalue needing to be a negative real number, say $-\lambda^{2}$, we can now obtain that Eq. (13) becomes

$$
\begin{aligned}
& \frac{X^{\prime \prime}(x)}{X(x)}=-\lambda^{2}, \\
& -\frac{Y^{\prime \prime}(y)}{Y(y)}+\frac{T^{\prime \prime}(t)}{T(t)}=-\lambda^{2} .
\end{aligned}
$$

We get the solution of Eq. (15) as $X(x)=C_{1} \cos (\lambda x)+C_{2} \sin (\lambda x)$, where $C_{1}, C_{2}$ are arbitrary constants. According to the boundary condition $X(0)=0$, we have $C_{1}=0$. Then $X(x)=$ $C_{2} \sin (\lambda x)$. The boundary condition $X(1)=0$ gives $\lambda=n \pi$, we then have

$$
X_{n}(x)=C_{2} \sin (n \pi x),
$$

where $n=1,2,3, \ldots$. Since the eigenvalue of Eq. (16) is a negative real number, say $-\mu^{2}$, we have

$$
\begin{aligned}
& \frac{Y^{\prime \prime}(y)}{Y(y)}=-\mu^{2}, \\
& \frac{T^{\prime \prime}(t)}{T(t)}+\lambda^{2}=-\mu^{2} .
\end{aligned}
$$

We then have the solutions $Y(y)=C_{3} \cos (\mu y)+C_{4} \sin (\mu y)$ and $T(t)=C_{5} \cos \left(t \sqrt{\lambda^{2}+\mu^{2}}\right)+$ $C_{6} \sin \left(t \sqrt{\lambda^{2}+\mu^{2}}\right)$, where $C_{3}, C_{4}, C_{5}, C_{6}$ are arbitrary constants. According to the boundary conditions $Y(0)=0$ and $Y^{\prime}(1)=0$, we have $C_{3}=0$ and $\mu=(2 m-1) \frac{\pi}{2}$. Thus,

$$
Y_{m}(y)=C_{4} \sin \left((2 m-1) \frac{\pi}{2} y\right)
$$


where $m=1,2,3, \ldots$. Then $Y(1)=g(x, t)=C_{4} \sin \left((2 m-1) \frac{\pi}{2}\right)$, we obtain $C_{4}=(-1)^{-m-1} g(x, t)$. We get

$$
Y_{m}(y)=(-1)^{-m-1} g(x, t) \sin \left((2 m-1) \frac{\pi}{2} y\right) .
$$

Since $\lambda=n \pi$ and $\mu=(2 m-1) \frac{\pi}{2}$, we then have

$$
T_{m n}(t)=C_{5} \cos \left(\pi t \sqrt{n^{2}+\left(\frac{2 m-1}{2}\right)^{2}}\right)+C_{6} \sin \left(\pi t \sqrt{n^{2}+\left(\frac{2 m-1}{2}\right)^{2}}\right) .
$$

According to Eqs. (17), (21) and (22), the solution of our problem can be written in the form

$$
\begin{aligned}
d_{m n}(x, y, t)= & X_{n}(x) Y_{m}(y) T_{m n}(t) \\
= & \sin (n \pi x)(-1)^{-m-1} g(x, t) \sin \left((2 m-1) \frac{\pi}{2} y\right) \\
& \times\left(A_{m n} \cos \left(\pi t \sqrt{n^{2}+\left(\frac{2 m-1}{2}\right)^{2}}\right)\right. \\
& \left.+B_{m n} \sin \left(\pi t \sqrt{n^{2}+\left(\frac{2 m-1}{2}\right)^{2}}\right)\right),
\end{aligned}
$$

where $A_{m n}=C_{2} C_{5}$ and $B_{m n}=C_{2} C_{6}$ are arbitrary constants and for all $m, n=1,2,3, \ldots$. By using the superposition principle, Eq. (24) becomes

$$
\begin{aligned}
d(x, y, t)= & \sum_{m=1}^{\infty} \sum_{n=1}^{\infty} d_{m n}(x, y, t) \\
= & \sum_{m=1}^{\infty} \sum_{n=1}^{\infty} \sin (n \pi x)(-1)^{-m-1} g(x, t) \sin \left((2 m-1) \frac{\pi}{2} y\right) \\
& \times\left(A_{m n} \cos \left(\pi t \sqrt{n^{2}+\left(\frac{2 m-1}{2}\right)^{2}}\right)\right. \\
& \left.+B_{m n} \sin \left(\pi t \sqrt{n^{2}+\left(\frac{2 m-1}{2}\right)^{2}}\right)\right)
\end{aligned}
$$

where $A_{m n}$ and $B_{m n}$ are fixed by the value of $d(x, y, 0)=f_{1}(x, y)$ and $\frac{\partial d}{\partial t}(x, y, 0)=f_{2}(x, y)$, respectively.

\section{Application to open uniform reservoir}

We consider an open uniform reservoir with dimension $3.2 \times 3.2 \mathrm{~km}(l=3.2 \mathrm{~km})$ and the constant depth $h=1 \mathrm{~m}$. Initially, the water in the reservoir is assumed to be motionless $u=0, v=0$ and the water elevation is specified by $\zeta(x, y, 0)=2 \sin (\pi x) \sin \left(\frac{\pi}{2} y\right)$ and $\frac{\partial \zeta}{\partial t}(x, y, 0)=-\sin (2 \pi x) \sin \left(\frac{\pi}{2} y\right)$. The elevation of water along the northern boundary is distributed by $\zeta(x, l, t)=2 \sin (\pi x) \cos (t)$. The open boundary condition is assumed to be 
$d(x, 1, t)=g(x, t)=2 \sin (\pi x) \cos (t)$. Eq. (26) becomes

$$
\begin{aligned}
d(x, y, t)= & \sum_{m=1}^{\infty} \sum_{n=1}^{\infty} \sin (n \pi x) 2(-1)^{-m-1} \sin (\pi x) \cos (t) \sin \left((2 m-1) \frac{\pi}{2} y\right) \\
& \times\left(A_{m n} \cos \left(\pi t \sqrt{n^{2}+\left(\frac{2 m-1}{2}\right)^{2}}\right)\right. \\
& \left.+B_{m n} \sin \left(\pi t \sqrt{n^{2}+\left(\frac{2 m-1}{2}\right)^{2}}\right)\right) .
\end{aligned}
$$

The initial conditions are assumed to be $d(x, y, 0)=f_{1}(x, y)=2 \sin (\pi x) \sin \left(\frac{\pi}{2} y\right)$, $\frac{\partial d}{\partial t}=$ $f_{2}(x, y)=-\sin (2 \pi x) \sin \left(\frac{\pi}{2} y\right)$. Thus Eq. (27) becomes

$$
\begin{aligned}
& \sum_{m=1}^{\infty} \sum_{n=1}^{\infty} A_{m n} \sin (n \pi x) 2(-1)^{-m-1} \sin (\pi x) \sin \left((2 m-1) \frac{\pi}{2} y\right) \\
& \quad=2 \sin (\pi x) \sin \left(\frac{\pi}{2} y\right)
\end{aligned}
$$

and

$$
\begin{aligned}
& \sum_{m=1}^{\infty} \sum_{n=1}^{\infty} B_{m n}\left(\pi \sqrt{n^{2}+\left(\frac{2 m-1}{2}\right)^{2}}\right) \sin (n \pi x) 2(-1)^{-m-1} \sin (\pi x) \sin \left((2 m-1) \frac{\pi}{2} y\right) \\
& \quad=-\sin (2 \pi x) \sin \left(\frac{\pi}{2} y\right) .
\end{aligned}
$$

We will consider the coefficients $A_{m n}$ by comparing coefficients and using the mathematical induction on the finite sum of Eq. (28) as follows:

$$
2 \sin (\pi x) \sin \left(\frac{\pi}{2} y\right) \simeq \sum_{m=1}^{M} \sum_{n=1}^{N} A_{m n} \sin (n \pi x) 2(-1)^{-m-1} \sin (\pi x) \sin \left((2 m-1) \frac{\pi}{2} y\right)
$$

By comparing their coefficients in Eq. (30), we can see that if $M=1$ and $N=1$,

$$
A_{11}=\frac{1}{\sin (\pi x)}
$$

where $\sin (\pi x) \neq 0$. Assume that $A_{11}=\frac{1}{\sin (\pi x)}$, where $M, N=k$ and $\sin (\pi x) \neq 0$. If $M=k+1$ and $N=k+1$, then

$$
\begin{aligned}
2 \sin (\pi x) \sin \left(\frac{\pi}{2} y\right) \\
=A_{11} \sin (\pi x) 2 \sin (\pi x) \sin \left(\frac{\pi}{2} y\right)+A_{12} \sin (2 \pi x) 2 \sin (\pi x) \sin \left(\frac{\pi}{2} y\right) \\
\quad+\cdots+A_{1 k} \sin (k \pi x) 2 \sin (\pi x) \sin \left(\frac{\pi}{2} y\right) \\
\quad+A_{1(k+1)} \sin ((k+1) \pi x) 2 \sin (\pi x) \sin \left(\frac{\pi}{2} y\right)
\end{aligned}
$$




$$
\begin{aligned}
& +A_{21} \sin (\pi x) 2(-1) \sin (\pi x) \sin \left(\frac{3 \pi}{2} y\right) \\
& +A_{22} \sin (2 \pi x) 2(-1) \sin (\pi x) \sin \left(\frac{3 \pi}{2} y\right) \\
& +\cdots+A_{2 k} \sin (k \pi x) 2(-1) \sin (\pi x) \sin \left(\frac{3 \pi}{2} y\right) \\
& +A_{2(k+1)} \sin ((k+1) \pi x) 2(-1) \sin (\pi x) \sin \left(\frac{3 \pi}{2} y\right) \\
& +\cdots+A_{(k+1) 1} \sin (\pi x) 2(-1)^{-k-2} \sin (\pi x) \sin \left(\frac{(2(k+1)-1) \pi}{2} y\right) \\
& +A_{(k+1) 2} \sin (2 \pi x) 2(-1)^{-k-2} \sin (\pi x) \sin \left(\frac{(2(k+1)-1) \pi}{2} y\right) \\
& +\cdots+A_{(k+1) k} \sin (k \pi x) 2(-1)^{-k-2} \sin (\pi x) \sin \left(\frac{(2(k+1)-1) \pi}{2} y\right) \\
& +A_{(k+1)(k+1)} \sin ((k+1) \pi x) 2(-1)^{-k-2} \sin (\pi x) \sin \left(\frac{(2(k+1)-1) \pi}{2} y\right) .
\end{aligned}
$$

Since $\sin (n \pi x) \neq 0$ and $\sin \left(\frac{(2 m-1) \pi}{2} y\right) \neq 0$ for all $x, y \in(0,1)$, substituting Eq. (31) into Eq. (32) and comparing the coefficients in Eq. (32), we can see that $A_{12}, A_{13}, \ldots, A_{1(k+1)}=0$, $A_{21}, A_{22}, \ldots, A_{2(k+1)}=0, \ldots, A_{(k+1) 1}, A_{(k+1) 2}, \ldots, A_{(k+1)(k+1)}=0$, except $A_{11} \neq 0$. We get

$$
A_{11}=\frac{1}{\sin (\pi x)} .
$$

Thus, Eq. (30) holds for $M=k+1$ and $N=k+1$, the proof of the induction step is completed. Similarly, we also consider the coefficients $B_{m n}$ by comparing coefficients and the mathematical induction on the finite sum of Eq. (29) as follows:

$$
\begin{aligned}
-\sin (2 \pi x) \sin \left(\frac{\pi}{2} y\right) \simeq & \sum_{m=1}^{M} \sum_{n=1}^{N} B_{m n}\left(\pi \sqrt{n^{2}+\left(\frac{2 m-1}{2}\right)^{2}}\right) \sin (n \pi x) 2(-1)^{-m-1} \\
& \times \sin (\pi x) \sin \left((2 m-1) \frac{\pi}{2} y\right)
\end{aligned}
$$

If $M=1$ and $N=2$, then

$$
\begin{aligned}
-\sin (2 \pi x) \sin \left(\frac{\pi}{2} y\right)= & B_{11}\left(\pi \sqrt{1^{2}+\left(\frac{1}{2}\right)^{2}}\right) \sin (\pi x) 2 \sin (\pi x) \sin \left(\frac{\pi}{2} y\right) \\
& +B_{12}\left(\pi \sqrt{2^{2}+\left(\frac{1}{2}\right)^{2}}\right) \sin (2 \pi x) 2 \sin (\pi x) \sin \left(\frac{\pi}{2} y\right) .
\end{aligned}
$$

By comparing their coefficients in Eq. (35), we get

$$
B_{12}=-\frac{1}{2 \pi \sqrt{4.25} \sin (\pi x)},
$$


where $B_{11}=0$ and $\sin (\pi x) \neq 0$. Assume that $B_{12}=-\frac{1}{2 \pi \sqrt{4.25} \sin (\pi x)}$, where $M, N=k$ and $\sin (\pi x) \neq 0$. If $M, N=k+1$, then

$$
\begin{aligned}
& -\sin (2 \pi x) \sin \left(\frac{\pi}{2} y\right) \\
& =B_{11}(\pi \sqrt{1.25}) \sin (\pi x) 2 \sin (\pi x) \sin \left(\frac{\pi}{2} y\right) \\
& +B_{12}(\pi \sqrt{4.25}) \sin (2 \pi x) 2 \sin (\pi x) \sin \left(\frac{\pi}{2} y\right) \\
& +\cdots+B_{1 k}\left(\pi \sqrt{k^{2}+0.25}\right) \sin (k \pi x) 2 \sin (\pi x) \sin \left(\frac{\pi}{2} y\right) \\
& +B_{1(k+1)}\left(\pi \sqrt{(k+1)^{2}+0.25}\right) \sin ((k+1) \pi x) 2 \sin (\pi x) \sin \left(\frac{\pi}{2} y\right) \\
& +B_{21}(\pi \sqrt{1+2.25}) \sin (\pi x) 2(-1) \sin (\pi x) \sin \left(\frac{3 \pi}{2} y\right) \\
& +B_{22}\left(\pi \sqrt{2^{2}+2.25}\right) \sin (2 \pi x) 2(-1) \sin (\pi x) \sin \left(\frac{3 \pi}{2} y\right) \\
& +\cdots+B_{2 k}\left(\pi \sqrt{k^{2}+2.25}\right) \sin (k \pi x) 2(-1) \sin (\pi x) \sin \left(\frac{3 \pi}{2} y\right) \\
& +B_{2(k+1)}\left(\pi \sqrt{(k+1)^{2}+2.25}\right) \sin ((k+1) \pi x) 2(-1) \sin (\pi x) \sin \left(\frac{3 \pi}{2} y\right) \\
& +\cdots+B_{(k+1) 1}\left(\pi \sqrt{1+\left(\frac{2(k+1)-1}{2}\right)^{2}}\right) \sin (\pi x) 2(-1)^{-k-2} \\
& \times \sin (\pi x) \sin \left(\frac{(2(k+1)-1) \pi}{2} y\right) \\
& +\cdots+B_{(k+1) 1}\left(\pi \sqrt{1+\left(\frac{2(k+1)-1}{2}\right)^{2}}\right) \sin (\pi x) 2(-1)^{-k-2} \\
& \times \sin (\pi x) \sin \left(\frac{(2(k+1)-1) \pi}{2} y\right) \\
& +B_{(k+1) 2}\left(\pi \sqrt{2^{2}+\left(\frac{2(k+1)-1}{2}\right)^{2}}\right) \sin (2 \pi x) 2(-1)^{-k-2} \\
& \times \sin (\pi x) \sin \left(\frac{(2(k+1)-1) \pi}{2} y\right) \\
& +\cdots+B_{(k+1) k}\left(\pi \sqrt{k^{2}+\left(\frac{2(k+1)-1}{2}\right)^{2}}\right) \sin (k \pi x) 2(-1)^{-k-2} \\
& \times \sin (\pi x) \sin \left(\frac{(2(k+1)-1) \pi}{2} y\right) \\
& +B_{(k+1)(k+1)}\left(\pi \sqrt{(k+1)^{2}+\left(\frac{2(k+1)-1}{2}\right)^{2}}\right) \sin ((k+1) \pi x) 2(-1)^{-k-2} \\
& \times \sin (\pi x) \sin \left(\frac{(2(k+1)-1) \pi}{2} y\right) .
\end{aligned}
$$


Since $\sin (n \pi x) \neq 0$ and $\sin \left(\frac{(2 m-1) \pi}{2} y\right) \neq 0$ for all $x, y \in(0,1)$, we can see that $B_{11}, B_{13}, B_{14}, \ldots$, $B_{1(k+1)}=0, B_{21}, B_{22}, \ldots, B_{2(k+1)}=0, \ldots, B_{(k+1) 1}, B_{(k+1) 2}, \ldots, B_{(k+1)(k+1)}=0$, except $B_{12} \neq 0$. We get

$$
B_{12}=\frac{1}{2 \pi \sqrt{4.25} \sin (\pi x)} .
$$

Thus, Eq. (34) holds for $M=k+1$ and $N=k+1$, the proof of the induction step is completed. Therefore, an analytical solution $d(x, y, t)$ of Eq. (27) with given initial and boundary conditions becomes

$$
\begin{aligned}
d(x, y, t)= & 2 \sin (\pi x) \cos (t) \sin \left(\frac{\pi}{2} y\right) \cos (\pi t \sqrt{1.25}) \\
& -\frac{1}{\pi \sqrt{4.25}} \sin (2 \pi x) \cos (t) \sin \left(\frac{\pi}{2} y\right) \sin (\pi t \sqrt{4.25})
\end{aligned}
$$

Table 1 Water elevation $\zeta(x, y, t)(\mathrm{m})$ at $t=2 \mathrm{hrs} 50 \mathrm{~min}$

\begin{tabular}{rlllllllll}
\hline $\boldsymbol{y} \backslash \boldsymbol{x}$ & $\mathbf{3 2 0}$ & $\mathbf{6 4 0}$ & $\mathbf{9 6 0}$ & $\mathbf{1 , 2 8 0}$ & $\mathbf{1 , 6 0 0}$ & $\mathbf{1 , 9 2 0}$ & $\mathbf{2 , 2 4 0}$ & $\mathbf{2 , 5 6 0}$ & $\mathbf{2 , 8 8 0}$ \\
\hline 320 & 0.1729 & 0.2620 & 0.3174 & 0.3352 & 0.3177 & 0.2716 & 0.2055 & 0.1272 & 0.0430 \\
640 & 0.2835 & 0.4295 & 0.5203 & 0.5495 & 0.5209 & 0.4453 & 0.3368 & 0.2085 & 0.0704 \\
960 & 0.3871 & 0.5864 & 0.7104 & 0.7503 & 0.7112 & 0.6080 & 0.4599 & 0.2847 & 0.0962 \\
1,280 & 0.4811 & 0.7289 & 0.8830 & 0.9326 & 0.8840 & 0.7557 & 0.5716 & 0.3538 & 0.1195 \\
1,600 & 0.5633 & 0.8534 & 1.0338 & 1.0919 & 1.0350 & 0.8848 & 0.6692 & 0.4143 & 0.1400 \\
1,920 & 0.6316 & 0.9570 & 1.1592 & 1.2243 & 1.1605 & 0.9922 & 0.7504 & 0.4646 & 0.1570 \\
2,240 & 0.6844 & 1.0369 & 1.2561 & 1.3266 & 1.2575 & 1.0751 & 0.8131 & 0.5034 & 0.1701 \\
2,560 & 0.7203 & 1.0913 & 1.3220 & 1.3963 & 1.3235 & 1.1315 & 0.8558 & 0.5298 & 0.1790 \\
2,880 & 0.7385 & 1.1189 & 1.3554 & 1.4315 & 1.3569 & 1.1600 & 0.8774 & 0.5432 & 0.1835 \\
\hline
\end{tabular}

Figure 2 Water elevation $\zeta(x, y, t)$, where $0 \leq t \leq 170(\mathrm{~min})$ at the center point of an open uniform reservoir.

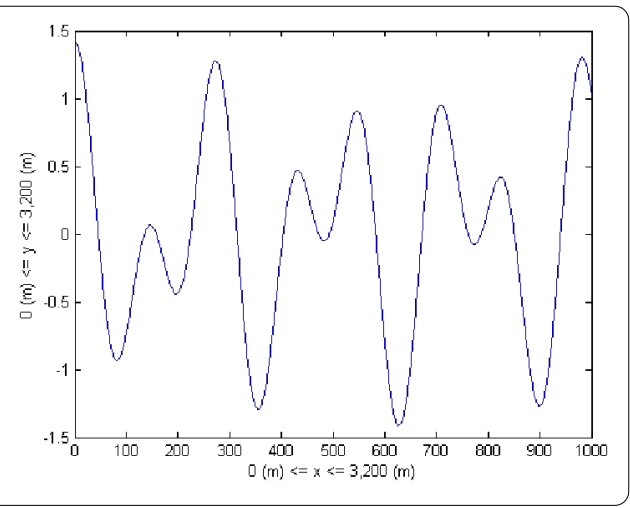

Figure 3 Surface plot of water elevation $\zeta(x, y, t)$ at $t=170$ ( $\mathrm{min}$ ) over an open uniform reservoir.

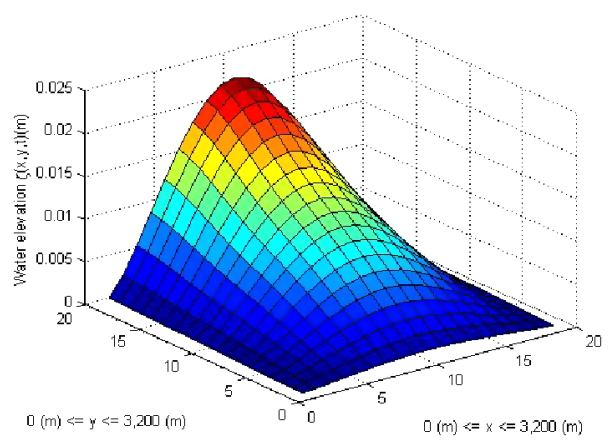


for all ( $x, y, t) \in \Omega \times[0, T]$. The solution of water wave elevations (tidal elevation) by using Eq. (39) is shown in Table 1 and Figures 2-3.

\section{Conclusions}

A two-dimensional hydrodynamic model for describing the elevation of water wave in an open uniform reservoir is derived and presented. The separation of variables method is employed to find an analytical solution to the model.

\section{Acknowledgements}

This paper is supported by the Centre of Excellence in Mathematics, the Commission on Higher Education, Thailand. The authors greatly appreciate valuable comments received from the reviewers.

\section{Competing interests}

The authors declare that they have no competing interests.

\section{Authors' contributions}

KT wrote the computer codes for the theoretical analysis, data acquisition, constructed the software and calibrated it and wrote the paper. JK contributed equally to the theoretical analysis, data acquisition, the interpretation of the solutions. Both authors read and approved the final manuscript.

\section{Publisher's Note}

Springer Nature remains neutral with regard to jurisdictional claims in published maps and institutional affiliations.

Received: 1 February 2017 Accepted: 12 May 2017 Published online: 25 May 2017

\section{References}

1. Pochai, N, Tangmanee, S, Crane, LJ, Miller, JJH: A mathematical model of water pollution control using the finite element method. In: Proceedings in Applied Mathematics and Mechanics, Berlin, 27-31 March 2006 (2006)

2. Tabuenca, P, Vila, J, Cardona, J, Samartin, A: Finite element simulation of dispersion in the Bay of Santander. Adv. Eng. Softw. 28, 313-332 (1997)

3. Pochai, $\mathrm{N}$, Tangmanee, S, Crane, LJ, Miller, JJH: A water quality computation in the uniform channel. J. Interdiscip. Math. 11, 803-814 (2008)

4. Pochai, $\mathrm{N}$ : A numerical computation of non-dimensional form of stream water quality model with hydrodynamic advection-dispersion-reaction equations. Nonlinear Anal. Hybrid Syst. 13, 666-673 (2009)

5. Pochai, N: A numerical computation of non-dimensional form of a nonlinear hydrodynamic model in a uniform reservoir. Nonlinear Anal. Hybrid Syst. 3, 463-466 (2009)

\section{Submit your manuscript to a SpringerOpen ${ }^{\circ}$ journal and benefit from:}

- Convenient online submission

Rigorous peer review

- Immediate publication on acceptance

- Open access: articles freely available online

- High visibility within the field

- Retaining the copyright to your article 